\title{
Apresentação física de produtos em projetos de produtos alimentícios: proposição de um processo para a especificação
}

RESUMO

Thays J. Perassoli Boiko

thaysperassoli@bol.com.br

Universidade Estadual do Paraná (UNESPAR), Campo Mourão, Paraná, Brasil

Letícia Fernanda Alves Pires piresleticia@hotmail.com Universidade Estadual do Paraná (UNESPAR), Campo Mourão, Paraná, Brasil

\section{Fabiane Avanzi Rezende} rezende.fabiane@hotmail.com Universidade Estadual do Paraná (UNESPAR), Campo Mourão, Paraná, Brasil

Vander Luiz da Silva vander-luiz@hotmail.com Universidade Estadual do Paraná (UNESPAR), Campo Mourão, Paraná, Brasil

\section{Rayane Carla Scheffer} rayane.scheffer@hotmail.com
Universidade Estadual do Paraná (UNESPAR), Campo Mourão, Paraná, Brasil
O setor de alimentos está cada vez mais competitivo e seus consumidores estão cada vez mais críticos e exigentes. Para obter vantagens competitivas, as empresas precisam estar capacitadas no desenvolvimento de Projetos de Produtos (PP). Entre as tarefas de um PP está a criação de especificações para os atributos do produto. Um atributo muito importante e complexo é a Apresentação Física, que define outros atributos e tem alto impacto mercadológico, logístico (armazenagem e distribuição), na produção e custos do produto. Os objetivos desta pesquisa foram investigar o atributo Apresentação Física, e propor um processo para sua especificação em PP alimentícios. O fluxograma, detalhamento e/ou fundamentação de cada etapa e uma exemplificação da aplicação do processo são apresentados. A metodologia de pesquisa é comentada. Na revisão de literatura não foram encontrados trabalhos apresentando processos para a especificação de Apresentação Física. A exemplificação da aplicação do processo apresenta simplicidade e flexibilidade, o que o torna aplicável na especificação de Aprensentação Física em PP realizado em pequenas e médias empresas, e em PP realizados por meio de Engenharia Simultânea. O processo fornece informações necessárias à realização de outras etapas em um PP. Sugere-se a aplicação do processo em outros casos e sua adaptação para a especificação de outros atributos do produto.

PALAVRAS-CHAVE: Desenvolvimento de produtos. Atributos de produto. Configuração física. 


\section{INTRODUÇÃO}

O setor de alimentos tem se tornado cada vez mais competitivo. Os consumidores estão cada vez mais críticos e exigentes em relação à qualidade, valor, aparência e funcionamento dos produtos (MCLLVEE,1994). Neste setor, as grandes inovações ocorrem nos alimentos semiprontos, pois os consumidores desejam, cada vez mais, reduzir o tempo com o preparo de refeições (GOUVEIA, 2006).

Para obter vantagens competitivas, nas empresas, é necessário desenvolver novos produtos, de forma eficiente, com custos viáveis e adequados às necessidades dos consumidores (ZUIN et al., 2004). Desta forma, são exigidas maiores capacitações das empresas e de profissionais, no desenvolvimento de projetos de novos produtos.

Define-se Projeto de Produto (PP) o processo de negócio cujas tarefas são: a) analisar as necessidades e expectativas dos stakeholders e criar as especificações para o produto e/ou serviço (ou linha de produtos e/ou serviços), e; transmitir todas as informações sobre o projeto e o produto para todas as áreas funcionais da empresa (MOREIRA, 2000).

Entre as especificações para o produto e/ou serviço (ou linha de produtos e/ou serviços) estão as especificações dos Atributos do Produto, que fazem parte do Desenvolvimento do Produto e Teste do Conceito do Produto.

Um atributo bastante importante e complexo é o atributo Apresentação Física (AF). Pois, esse define vários outros atributos do produto e/ou serviço, desde sua classificação, conforme a legislação, passando por sua embalagem, estruturação em linhas de produtos e chegando na definição de seu preço de mercado. Logo, tem grande impacto mercadológico. Além disso, tem também impacto na logística (armazenagem e distribuição), produção e custos do produto.

Os objetivos da pesquisa, aqui relatada, foram investigar o atributo AF de Produtos, sua importância e como especificá-lo em PP alimentícios, buscando propor um Processo para a especificação da AF de Produtos em PP alimentícios.

O Processo proposto foi desenvolvido a partir da sistematização detalhada do Processo utilizado, desde 2008, nos projetos realizados em uma Disciplina de PP (BOIKO, 2013).

Como exemplificação da aplicação do Processo proposto, apresentam-se os resultados de sua aplicação na especificação da AF dos produtos desenvolvidos em um PP de linhas de minipizzas prontas (Minipizza destinada para o consumo individual, Porções de minipizza e Minipizza para festas) realizado em 2013, como requisito de avaliação da já referida Disciplina (REZENDE et al., 2013).

Embora o Processo proposto tenha sido inicialmente aplicado neste projeto, pode ser utilizado para especificar a AF em PP de outros tipos de alimentos.

O artigo está estruturado em cinco seções. Inicialmente a pesquisa é contextualizada e seus objetivos são apresentados. Em seguida, trata-se do atributo AF. Depois, tem-se a metodologia. Na quarta seção, apresenta-se a aplicação e exemplificação do Processo proposto. Por fim, apresentam-se as considerações finais. 


\section{Apresentação Física de produtos alimentícios}

A Apresentação Física (AF), também denominada de Configuração Física (COBRA, 1992) e Forma Física (ANVISA, 2005), diz respeito ao formato ou forma do produto, ao estado físico e/ou sua consistência e/ou textura do produto, suas medidas (peso e tamanho), às unidades de produto na embalagem e à $A F$ da embalagem (formato ou forma, tamanho e apresentação de textura na embalagem).

Neste contexto, é um atributo que afeta os aspectos logísticos (armazenagem e distribuição) e de produção do produto, e, consequentemente seus custos.

Além disso, é um dos meios pelo qual é possível estabelecer uma relação entre o produto e o consumidor, no nível sensorial, através dos sentidos da visão, tato e paladar (GULLO; PINHEIRO, 2011), sendo assim, um atributo importante do ponto de vista mercadológico.

Na especificação da AF de um produto, deve-se levar em consideração os fatores que influenciam o comportamento de compra dos consumidores, os atributos e percepções do produto, a legislação para este produto e os produtos concorrentes, conforme apresenta a Figura 1

Figura 1 - Processo para a especificação da Apresentação Física de Produtos em Projeto de Produtos alimentícios

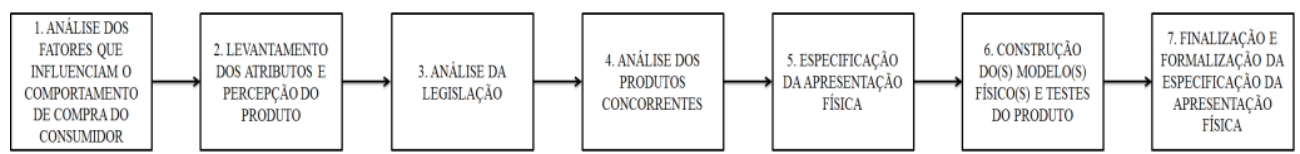

Fonte: Elaborado pelos autores, com informações obtidas em Boiko (2013).

As etapas do processo apresentado na Figura 1 estão detalhadas no Apêndice 1.

Salienta-se que o Processo é flexível, pois as etapas podem não ser realizadas, dependendo da disponibilidade de tempo, recursos e informações ou ainda da necessidade ou não de realização. Além disso, podem ser acrescentadas etapas e subetapas, conforme a necessidade de cada projeto.

As etapas e subetapas do processo para especificação da Apresentação Física apresentam relações de precedência outras não. Assim, estas podem ser adiantadas, caso exista à necessidade de fornecimento de informações à outras etapas do desenvolvimento do projeto.

Cada Etapa do Processo proposto é detalhada e/ou fundamentada nas subseções seguintes. 
Os fatores que influenciam o comportamento de compra dos consumidores correspondem aos fatores culturais, sociais, pessoais e psicológicos (RODRIGUES; JUPI, 2004).

a) Fatores culturais:

- Cultura: são os costumes das ações cotidianas, as tradições, as convecções e mitos (MOWEN; MINOR, 2003), envolvendo valores e crenças criados pela sociedade, que são passados de geração a geração no ambiente familiar e reforçada pelas instituições (TEIXEIRA, 2010);

- Subcultura: é a identificação da forma que cada cultura é influenciada (nacionalidades, religiões, grupos sociais e regiões geográficas) (SHETH; MITTAL; NEWMAN, 2001), e;

- Classe social: são divisões relativamente homogêneas e duradouras de uma sociedade, que são ordenadas hierarquicamente, cujos membros compartilham comportamentos similares, valores e interesses (KOTLER, 1998).

b) Fatores sociais:

- Grupos de referência: são os grupos de pessoas que exercem influência nos pensamentos, sentimentos e nos comportamentos do indivíduo (CHURCHILL; PETER, 2000), sendo este membro ou não de um grupo de pessoas (SCHIFFMAN; KANUK, 2000);

- Família: é o grupo com o qual o indivíduo interage de maneira contínua e informal (SCARABOTO, 2006), e;

- Papéis e posições sociais: são as atividades que se espera que um indivíduo desempenhe. As posições sociais estão relacionadas com o status do indivíduo na sociedade (SHIMOYAMA; ZELA, 2002).

c) Fatores pessoais: correspondem às características particulares dos indivíduos, ou seja, os momentos e convivências a que são expostos (KOTLER, 1998 e SOUZA, 2012).

- Idade e estágio de ciclo de vida e os ciclos de vida familiar (CHURCHILL; PETER, 2000);

- Ocupação: atividade e função (SOUSA, 2012) ou profissão que o indivíduo exerce (KOTLER, 1998);

- Condições econômicas: renda disponível, poupança e patrimônio, condições de crédito e atitudes em relação às despesas versus poupança (KOTLER, 1998);

- Estilo de vida: padrão de vida, manifestado pelo jeito como os indivíduos vivem, utilizam seu tempo e gastam seu dinheiro por suas atividades, interesses e/ou opiniões (GREWAL; LEVY, 2011), e;

- Personalidade e autoconceito: a personalidade é o conjunto de múltiplos componentes que incluem os valores, atitudes, crenças, motivos, intenções, preferências, opiniões, interesses, preconceitos e normas culturais do indivíduo (RICHERS, 1984). O autoconceito se refere às características psicológicas, como autoconfiança, autonomia, adaptabilidade e as relações com o ambiente, que influencia as escolhas do indivíduo (KOTLER; KELLER, 2006).

d) Fatores psicológicos: 
- Motivação: “... é uma necessidade que está pressionando suficientemente para levar uma pessoa a agir" (KOTLER, 1998, p. 173), sendo a força interna dos indivíduos que os leva à agir, quando existe uma necessidade não satisfeita (SCHIFFMAN; KANUK, 2000);

- Percepção: é a forma ou processo pelo qual um indivíduo seleciona, organiza e interpreta as informações ou estímulos para formar um quadro significativo e coerente do mundo (KOTLER, 1998; SCHIFFMAN; KANUK, 2000);

- Aprendizagem: "é um processo pelo qual a experiência resulta nas mudanças no conhecimento, atitudes e/ou comportamentos dos indivíduos." (ENGEL; BLACKWELL; MINIARD, 2000, p. 335), e;

- Crenças e atitudes: "crença é um pensamento descritivo que uma pessoa sustenta sobre algo" (KOTLER, 1998, p. 176). Já atitude é a avaliação, percepção e tendência de uma pessoa em relação a um objeto (COBRA, 2001).

\section{Levantamento dos atributos e percepções do produto}

Os atributos do produto dizem respeito ao que é o produto, do que é feito, como funciona, qual seu apelo promocional, quais necessidades do consumidor ele atende (DANTAS, 1998), sua classificação, do ponto de vista do Marketing e à categoria e classe (ou subcategoria) nas quais o produto se enquadra.

Cobra (1997) classifica os produtos de consumo em produtos de conveniência, produtos de compra comparada e produtos de especialidade.

As percepções do produto envolvem diversos aspectos, como:

- Temperatura: frio ou calor;

- Luminosidade: brilhante; apagada ou fosca;

- Peso: leve ou pesado;

- Sabor: doce, salgado, azedo, cítrico, agridoce, picante ou apimentado;

- Textura: crocante; leve; fluida; macia; áspera ou cremosa (DANTAS, 1998);

- Satisfação pela posse ou uso do produto;

- Sentimentos causados pela posse ou uso do produto: confiança, segurança, status, alegria, saudade;

- Funcionalidade: a utilidade básica prevista para o produto (GULLO; PINHEIRO, 2011);

- Acessibilidade;

- Competência;

- Valor;

- Satisfação (STONE, 1992), e;

- Percepção de qualidade, que envolve a qualidade definida para o produto, sua imagem e quanto o consumidor está disposto a pagar por este produto. 
A Análise da Legislação inicia com o levantamento da classificação do produto na legislação, de acordo com a categoria e classe (ou subcategoria) deste produto e de acordo com os ingredientes utilizados no produto.

Em seguida, com base nesta classificação, são levantadas as implicações da legislação para a Apresentação Física do Produto.

A Análise da Legislação deve ser realizada nas publicações oficiais dos órgãos, ministérios, secretarias, agências, entidades ou associações, no nível internacional (caso o produto seja destinado à exportação), federal, estadual e municipal que regulamentam e/ou certificam a produção, armazenagem, transporte, distribuição, comercialização e consumo do produto.

\section{Análise dos produtos concorrentes}

O termo produtos concorrentes significa produtos iguais, similares ou parecidos e produtos substitutos (COBRA, 1997).

$\mathrm{Na}$ Análise dos produtos concorrentes deve-se:

- Definir a categoria do produto;

- Definir sua classe (ou subcategoria);

- Levantar os atributos e as percepções dos produtos concorrentes;

- Analisar as Apresentações Físicas (AF) utilizadas pelos concorrentes: o estado físico e/ou a consistência e/ou textura; as unidades de produto nas embalagens; a AF das embalagens; o formato ou forma das embalagens; o tamanho das embalagens; a apresentação de texturas nas embalagens, e;

- Definir as estratégias de concorrência em termos de AF: a) utilizar a mesma AF dos concorrentes, no caso do produto ser classificado como produto de compra comparada; b) utilizar uma AF totalmente diferente da concorrência, no caso do produto de especialidade, e/ou; c) utilizar uma AF levemente diferente da concorrência, no caso dos produtos de compra comparada e de conveniência.

\section{Especificação da Apresentação Física}

A Especificação da Apresentação Física (AF) vai depender dos levantamentos, análises e decisões realizadas nas etapas anteriores do processo.

Em todos os itens que compõem a Especificação da AF é necessário levar em consideração as seguintes variáveis: os aspectos mercadológicos do produto; a legislação; aspectos e custos logísticos (armazenagem e distribuição); equipamentos e ferramentas, e; custos de produção. Deve-se considerar o item de AF mais viável, considerando todas estas variáveis.

A Especificação da AF envolve definir:

- Formato ou forma do produto;

- Estado físico e/ou sua consistência e/ou textura do produto; 
- Medidas do produto: peso do produto; tamanho do produto - dimensões (comprimento, largura e/ou altura); volume, e/ou; outros (conforme o formato ou forma do produto).

- Unidades de produto na embalagem;

- AF da embalagem: definir o formato ou forma da embalagem; definir o tamanho da embalagem - dimensões (comprimento, largura e/ou altura); volume, e/ou; outros (conforme o formato ou forma da embalagem), e;

- Apresentação de textura(s) na embalagem.

Quanto à textura, tanto no produto, quanto na embalagem, é possível fazer diversas combinações, proporcionando ao consumidor uma grande variedade de sensações (calor, frio, leveza, suavidade, entre outros) (LEYSER, 2012). Assim, na definição de texturas é preciso considerar a sensação que se deseja despertar no consumidor.

A AF da embalagem possibilita a diferenciação entre os produtos de um mesmo segmento de mercado (CAMPOS; NANTES, 1999).

O tamanho das embalagens está relacionado com o tamanho das famílias de produtos (FARIA; SOUZA, 2008).

\section{Construção do(s) Modelo(s) Físico(s) e Teste do Produto}

Depois de especificada a Apresentação Física (AF) do Produto passa-se para as etapas de construção do(s) modelo(s) físico(s) do produto e testes.

Os seguintes modelos físicos podem ser construídos:

- Mockups: qualquer modelo tridimensional de um produto ou de seus componentes, construído fisicamente, em escala natural (real), independente do material utilizado para construí-lo e que não precisa reproduzir, necessariamente, suas funções;

- Protótipos: qualquer modelo tridimensional de um produto ou de seus componentes, construído fisicamente, em escala natural (real), com materiais especificados em desenho, ou materiais com características semelhantes, capazes de reproduzir, necessariamente, suas funções e serem testados, e;

- Modelos: qualquer modelo tridimensional de um produto ou de seus componentes, construído fisicamente, em qualquer escala, precisamente com materiais especificados em desenho, e que não precisa reproduzir, necessariamente, suas funções (MACARRÃO JUNIOR, 2004).

A escolha de um ou outro modelo depende dos recursos físicos e financeiros disponíveis e das necessidades dos testes.

Construído(s) o(s) modelo(s) físico(s) passa-se para os testes, que irão apresentar as necessidades de adaptação da Apresentação Física (AF).

O Teste de Fabricação mostra o processo de fabricação do produto, os equipamentos e ferramental disponível e, consequentemente com qual AF é possível fabricar o produto.

O Teste de Custos mostra os custos logísticos (armazenagem e distribuição) e de produção do produto, conforme a AF especificada e os custos para diferentes 
AF do produto e da embalagem. Consultar Black (2008), para detalhes de como realizar.

O Teste com Consumidores mostra a opinião dos consumidores sobre os vários aspectos da $\mathrm{AF}$ do produto e de sua embalagem, mostrando o que é necessário mudar na AF para adaptar o produto e sua embalagem aos sentidos do consumidor. Consultar Cobra (1997) e Kotler (1998), para detalhes dos procedimentos de realização.

Ressalta-se que, a disponibilidade de tempo, recursos físicos e/ou financeiros determina quais testes serão ou não realizados.

\section{Finalização e formalização da Especificação da Apresentação Física}

Finalizada a etapa de adaptação da Apresentação Física (AF), conforme as necessidades levantadas nos testes, torna-se necessário finalizar e formalizar sua especificação. Nesta etapa, são gerados os documentos que detalham esta especificação em todos os seus aspectos, devendo conter fotos, desenhos, fluxogramas e/ou todas as descrições técnicas (itens de especificação detalhados na Etapa 5 do Processo proposto) necessárias ao desenvolvimento do Projeto do Produto e sua futura produção e distribuição.

\section{METODOLOGIA}

O método de abordagem utilizado na proposição do Processo de Especificação da Apresentação Física (AF) de produtos foi o qualitativo. A pesquisa utilizada para desenvolver o Processo, classifica-se, quanto aos fins, como descritiva, exploratória e metodológica e, quantos aos meios, como bibliográfica, dos tipos tradicional e virtual.

A pesquisa utilizada na aplicação do processo classifica-se, quanto aos fins, como aplicada, descritiva e metodológica e, quantos aos meios, como bibliográfica, dos tipos tradicional e virtual, pesquisa documental, estudo de caso, experimental e pesquisa participante.

A revisão de literatura focou-se na busca por trabalhos que tratassem de AF, utilizando além deste termo, as seguintes palavras-chaves: Configuração Física e Forma Física. Contudo, na literatura não foram encontrados trabalhos sobre AF em PP.

\section{APLICAÇÃO DO PROCESSO DE ESPECIFICAÇÃO DA APRESENTAÇÃO FÍSICA NO PROJETO DE PRODUTOS DAS LINHAS DE MINIPIZZAS PRONTAS}

Os Quadros 1, 2, 3, 4, 5, 6 e 7 apresentam-se os resultados da aplicação das Etapas 1 a 7, respectivamente, do processo proposto na especificação da Apresentação Física no Projeto de Produtos das Linhas de Minipizzas Prontas. 
Quadro 1 - Resultados da aplicação da Etapa 1 do processo proposto

\begin{tabular}{|c|c|c|}
\hline & & \\
\hline & & $\begin{array}{l}\text { RESULTADOS DA APLICAÇÃO NO } \\
\text { PROJETO DE PRODUTOS DAS } \\
\text { LINHAS DE MINIPIZZAS PRONTAS }\end{array}$ \\
\hline \multirow{3}{*}{$\begin{array}{c}1 \text { ANÁLISE DOS } \\
\text { FATORES QUE } \\
\text { INFLUENCIAM O } \\
\text { COMPORTAMENTO DE } \\
\text { COMPRA DOS } \\
\text { CONSUMIDORES }\end{array}$} & 1.3 ANÁLISE DOS FATORES PESSOAIS & ----- \\
\hline & 1.3.2 Análise da ocupação & $\begin{array}{l}\text { - Principalmente, pessoas que } \\
\text { moram sozinhas (BROGNOLI, } \\
\text { 2010). }\end{array}$ \\
\hline & 1.3.3 Análise das condições econômicas & $\begin{array}{l}\text { - Indivíduos das classes sociais B e } \\
\text { C. }\end{array}$ \\
\hline
\end{tabular}

Fonte: Elaborado pelos autores, com informações obtidas em Rezende et al. (2013).

Quadro 2 - Resultados da aplicação da Etapa 2 do processo proposto

\begin{tabular}{|c|c|c|}
\hline & & $\begin{array}{l}\text { RESULTADOS DA APLICAÇÃO NO } \\
\text { PROJETO DE PRODUTOS DAS } \\
\text { LINHAS DE MINIPIZZAS PRONTAS }\end{array}$ \\
\hline \multirow{7}{*}{$\begin{array}{l}2 \text { LEVANTAMENTO } \\
\text { DOS ATRIBUTOS E } \\
\text { PERCEPÇÕES DO } \\
\text { PRODUTO }\end{array}$} & $\begin{array}{l}\text { 2.1 LEVANTAMENTO DOS ATRIBUTOS DO } \\
\text { PRODUTO }\end{array}$ & ----- \\
\hline & 2.1.1 Análise do que é o produto & $\begin{array}{l}\text { - Alimento pronto e congelado; } \\
\text { - Alimento de consumo individual; } \\
\text { - Massa; } \\
\text { - Sobremesa ou lanche. }\end{array}$ \\
\hline & $\begin{array}{l}\text { 2.1.3 Análise de como funciona } o \\
\text { produto }\end{array}$ & $\begin{array}{l}\text { - Alimento de consumo rápido; } \\
\text { - Lanche; } \\
\text { - Sobremesa. }\end{array}$ \\
\hline & $\begin{array}{l}\text { 2.1.4 Análise do apelo promocional do } \\
\text { produto }\end{array}$ & \multirow{2}{*}{$\begin{array}{l}\text { - Praticidade; } \\
\text { - Rapidez no preparo; } \\
\text { - Diversidade de sabores. }\end{array}$} \\
\hline & $\begin{array}{l}\text { 2.1.5 Análise das necessidades do } \\
\text { consumidor }\end{array}$ & \\
\hline & $\begin{array}{l}\text { 2.1.6 Levantamento da classificação do } \\
\text { produto }\end{array}$ & - Produtos de conveniência. \\
\hline & $\begin{array}{l}\text { 2.1.7 Levantamento da categoria e da } \\
\text { classe (ou subcategoria) do produto }\end{array}$ & $\begin{array}{l}\text { - Categoria massas; } \\
\text { - Classe: massas prontas e } \\
\text { congeladas; } \\
\text { - Subclasse: lanches e sobremesas. }\end{array}$ \\
\hline
\end{tabular}

Fonte: Elaborado pelos autores, com informações obtidas em Rezende et al. (2013). 
Quadro 2 - Resultados da aplicação da Etapa 2 do Processo proposto

\begin{tabular}{|c|c|c|}
\hline & & \\
\hline & & $\begin{array}{c}\text { RESULTADOS DA APLICAÇÃO NO } \\
\text { PROJETO DE PRODUTOS DAS } \\
\text { LINHAS DE MINIPIZZAS } \\
\text { PRONTAS }\end{array}$ \\
\hline \multirow{4}{*}{$\begin{array}{l}2 \text { LEVANTAMENTO DOS } \\
\text { ATRIBUTOS E } \\
\text { PERCEPÇÕES DO } \\
\text { PRODUTO }\end{array}$} & $\begin{array}{l}\text { 2.2 LEVANTAMENTOS DAS PERCEPÇÕES } \\
\text { DO PRODUTO }\end{array}$ & ----- \\
\hline & $\begin{array}{l}\text { 2.2.4 Levantamentos das percepções de } \\
\text { sabor }\end{array}$ & - Doce \\
\hline & $\begin{array}{l}\text { 2.2.5 Levantamentos das percepções de } \\
\text { textura }\end{array}$ & $\begin{array}{l}\text { - Massa: crocante; } \\
\text { - Recheio: Cremosa. }\end{array}$ \\
\hline & $\begin{array}{l}\text { 2.2.9 Levantamentos das percepções de } \\
\text { acessibilidade do produto }\end{array}$ & $\begin{array}{l}\text { - Boa acessibilidade: produto } \\
\text { presente em todos os pontos de } \\
\text { venda adequados. }\end{array}$ \\
\hline
\end{tabular}

Fonte: Elaborado pelos autores, com informações obtidas em Rezende et al. (2013).

Quadro 3 - Resultados da aplicação da Etapa 3 do processo proposto

\begin{tabular}{|c|c|c|}
\hline & & $\begin{array}{l}\text { RESULTADOS DA APLICAÇÃO NO } \\
\text { PROJETO DE PRODUTOS DAS } \\
\text { LINHAS DE MINIPIZZAS PRONTAS }\end{array}$ \\
\hline \multirow{2}{*}{$\begin{array}{l}3 \text { ANÁLISE DA } \\
\text { LEGISLAÇÃO }\end{array}$} & $\begin{array}{l}\text { 3.1 LEVANTAMENTO DA CLASSIFICAÇÃO } \\
\text { DO PRODUTO NA LEGISLAÇÃO }\end{array}$ & $\begin{array}{l}\text { - Quanto ao produto: alimento } \\
\text { pronto e congelado; } \\
\text { - Quanto aos ingredientes: massa } \\
\text { alimentícia; produto com glútem; } \\
\text { produto com leite; produto com } \\
\text { frutas; produto com chocolate. }\end{array}$ \\
\hline & $\begin{array}{l}\text { 3.2 LEVANTAMENTO DAS IMPLICAÇÕES } \\
\text { DA LEGISLAÇÃO PARA A APRESENTAÇÃO } \\
\text { FÍSICA DESTE PRODUTO }\end{array}$ & - Nada específico. \\
\hline
\end{tabular}

Fonte: Elaborado pelos autores, com informações obtidas em Rezende et al. (2013).

Quadro 4 - Resultados da aplicação da Etapa 4 do processo proposto

\begin{tabular}{|c|c|c|}
\hline & & \multirow[b]{2}{*}{$\begin{array}{l}\text { RESULTADOS DA APLICAÇÃO NO } \\
\text { PROJETO DE PRODUTOS DAS } \\
\text { LINHAS DE MINIPIZZAS PRONTAS }\end{array}$} \\
\hline & & \\
\hline \multirow[t]{2}{*}{$\begin{array}{l}4 \quad \text { ANÁLISE } \\
\text { PRODUTOS } \\
\text { CONCORRENTES }\end{array}$} & $\begin{array}{l}4.3 \text { ANALISAR AS APRESENTAÇÕES } \\
\text { FISÍCAS (AF) UTILIZADAS PELOS } \\
\text { PRODUTOS CONCORRENTES }\end{array}$ & $\begin{array}{l}\text { - AF dos produtos: tamanhos de } \\
\text { massa variados; massas de textura } \\
\text { crocante; recheios cremosos; } \\
\text { massas de espessura média; } \\
\text { - AF das embalagens; formas } \\
\text { quadradas ou retangulares; } \\
\text { apresentação de diferentes } \\
\text { texturas na mesma embalagem. }\end{array}$ \\
\hline & $\begin{array}{l}\text { 4.4 DEFINIR AS ESTRATÉGIAS DE } \\
\text { CONCORRÊNCIA EM TERMOS DE AF }\end{array}$ & $\begin{array}{l}\text { - Utilizar as mesmas estratégias de } \\
\text { AF adotadas pelos concorrentes. }\end{array}$ \\
\hline
\end{tabular}


Fonte: Elaborado pelos autores, com informações obtidas em Rezende et al. (2013).

Quadro 5 - Resultados da aplicação da Etapa 5 do processo proposto

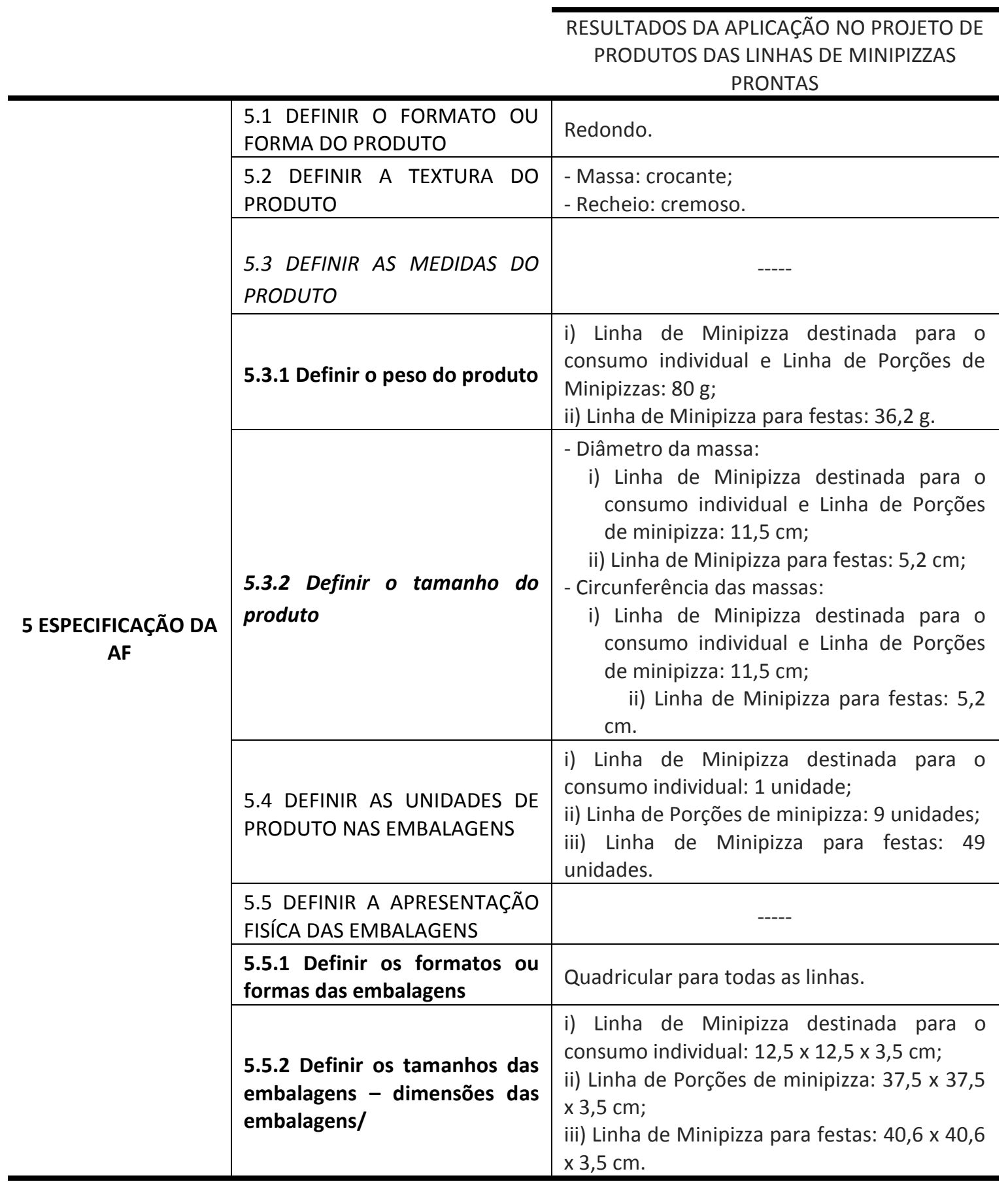

Fonte: Elaborado pelos autores, com informações obtidas em Rezende et al. (2013). 
Quadro 6 - Resultados da aplicação da Etapa 6 do Processo proposto

\begin{tabular}{|c|c|c|}
\hline & & $\begin{array}{l}\text { RESULTADOS DA APLICAÇÃO NO } \\
\text { PROJETO DE PRODUTOS DAS } \\
\text { LINHAS DE MINIPIZZAS PRONTAS }\end{array}$ \\
\hline \multirow{4}{*}{$\begin{array}{c}6 \text { CONSTRUÇÃO } \\
\text { DO(S) MODELO(S) } \\
\text { FÍSICO(S) E TESTES } \\
\text { DO PRODUTO }\end{array}$} & $\begin{array}{l}6.1 \text { DEFINIÇÃO DO(S) TIPO(S) DE } \\
M O D E L O(S) \text { FÍSICO(S) A UTILIZAR }\end{array}$ & - Protótipo \\
\hline & 6.3 TESTES DO PRODUTO & ----- \\
\hline & 6.3.2 Testes de Custos & $\begin{array}{l}\text { - Os custos para fabricar o produto } \\
\text { conforme Apresentação Física AF } \\
\text { especificada estão de acordo com o } \\
\text { preço que o consumidor está } \\
\text { disposto a pagar pelo produto. }\end{array}$ \\
\hline & 6.3.3 Testes com Consumidores & - A AF foi bem aceita \\
\hline
\end{tabular}

Fonte: Elaborado pelos autores, com informações obtidas em Rezende et al. (2013).

Quadro 7 - Resultados da aplicação da Etapa 7 do Processo proposto

RESULTADOS DA APLICAÇÃO NO PROJETO DE PRODUTOS DAS LINHAS DE MINIPIZZAS PRONTAS

\begin{tabular}{c|l} 
7 FINALIZAÇÃO E FORMALIZAÇÃO DA ESPECIFICAÇÃO DA & $\begin{array}{l}\text { - A formalização da AF foi } \\
\text { apresentada em um relatório } \\
\text { do Projeto do Produto }\end{array}$ \\
\hline
\end{tabular}

Fonte: Elaborado pelos autores, com informações obtidas em Rezende et al. (2013).

Como é possível visualizar, algumas etapas do processo não foram realizadas, pois a análise ou levantamento realizado nesta etapa não afeta o consumo de pizza no Brasil ou porque a especificação ou definição realizada nesta etapa não é necessária para os produtos em questão.

O teste de fabricação não foi realizado por falta de tempo e recursos. Já o teste com consumidores foi realizado por meio da técnica ensaio em laboratório, utilizando o método da análise sensorial e utilizando entrevistas estruturadas para coletar os dados necessários para medir a distribuição de preferência dos consumidores.

\section{CONCLUSÕES}

A Apresentação Física (AF) é um atributo bastante importante e complexo dos produtos, pois define vários outros, e tem impacto mercadológico, logístico, na produção e nos custos.

No entanto, não foram encontrados, na revisão de literatura realizada, trabalhos tratando deste assunto.

A exemplificação da aplicação do processo proposto, no trabalho realizado em uma Disciplina de Graduação, demonstra sua simplicidade, o que o torna 
aplicável para especificar a AF em Projetos de Produtos (PP) realizados em pequenas e médias empresas.

A exemplificação demonstra também a flexibilidade do Processo, pois etapas e subetapas podem ser realizadas ou não, de acordo com as necessidades, exigências e disponibilidades de cada projeto e conforme cada produto que está sendo projetado.

Ressalta-se que o Processo proposto fornece, também, informações necessárias à realização das seguintes tarefas de um PP: análise das necessidades e expectativas dos stakeholders, principalmente dos consumidores, e; criação de várias especificações para o produto.

O processo proposto pode ser aplicado em PP realizados por meio de Engenharia Simultânea.

Sugere-se a aplicação do processo em outros casos e sua adaptação para a especificação de outros atributos do produto, tais como Cor, Sabor, Aroma, Imagem, Linhas de Produto, por exemplo. 


\title{
Physical presentation of products in food products projects: proposition of a process for specification
}

\begin{abstract}
The food industry is increasingly competitive and its consumers are critical and demanding. To gain competitive advantages, the companies need to be skilled in the Projects Product (PP) developing. Among the tasks of a PP is the creation of specifications for the product attributes. A very important and complex attribute is the Physical Presentation, that define other attributes and it has high marketing costs and impact, logistic (storage and distribution), in the production and product costs. The research objectives were to investigate the Physical Presentation attribute, and propose a process for specification Physical Presentation in PP food. The flowchart, detailing and/or grounding for each phase, and an exemplification of the application process are presented. The research methodology is commented. In the literature review no papers presenting processes for the specification of Physical Presentation were found. The exemplification of the application process presents simplicity and flexibility, which makes it applicable in the specification of Physical Presentation in PP performed in small and medium enterprises, and in PP performed through Concurrent Engineering. The process provides information necessary to perform other steps on a PP. We suggest the application of the process to other cases and their adaptation to specify other attributes of the product.
\end{abstract}

KEYWORDS: Product development. Product attributes. Physical configuration. 


\section{REFERÊNCIAS}

ANVISA - AGÊNCIA NACIONAL DE VIGILÂNCIA SANITÁRIA. Passo a Passo para Novas Notificações de Produtos Cosméticos Grau 1: Gerência Geral de Cosméticos. Brasília, 2005.

BLACK, N. Projeto integrado de produtos: Planejamento, Concepção e Modelagem. Bauru: Manole, 2008.

BOIKO, T. J. P. Projeto de Produto: Apostila. Disciplina de Projeto do Produto, Curso de Engenharia de Produção Agroindustrial, Departamento de Engenharia de Produção, Campus de Campo Mourão, Universidade Estadual do Paraná (UNESPAR), Campo Mourão/PR, 2013.

BROGNOLI, M. L. Consumo de alimentos congelados por indivíduos frequentadores de supermercados. Criciúma, 2010. p. 12. Disponível em: <http://repositorio.unesc.net/bitstream/handle/1/146/Mariani\%20Lima\%20Brog noli.pdf?sequence=1>. Acesso em: 01 ago. 2013.

CAMPOS, H. C. M; NANTES, J. F. D. Embalagens convenientes: Uma estratégia na diferenciação dos produtos. In: XVIII CONGRESSO NACIONAL DE ENGENHARIA DE PRODUÇÃO. Rio de Janeiro: Abrepro, 1999.

CHURCHILL, G. A.; PETER, J. P. Marketing: criando valor para o cliente. São Paulo: Saraiva, 2000.

COBRA, M. H. N. Administração de Marketing. 2 ed. São Paulo: Atlas, 1992.

. Marketing Básico: Uma abordagem brasileira. 4 ed. São Paulo: Atlas, 1997.

Marketing de Turismo. São Paulo: Cobra, 2001.

Marketing e Moda. São Paulo: Cobra, 2008.

DANTAS, D. Parâmetros para a avaliação das embalagens de água sanitária.

Dissertação (Mestrado em Arquitetura e Urbanismo) - Universidade de São Paulo, USP. São Paulo, 1998.

ENGEL, J. C.; BLACKWELL, R. D.; MINIARD, P. W. Comportamento do Consumidor. 8 ed. Rio de Janeiro: LTC, 2000. 
FARIA, M. A.; SOUZA, C. V. A influência da embalagem no composto de marketing. In: IV CONGRESSO NACIONAL DE EXCELÊNCIA EM GESTÃO, 2008, Rio de Janeiro. Anais... Rio de Janeiro: 2008.

GOUVEIA, F. Indústria de alimentos: no caminho da inovação e de novos produtos. Revista inovação Uniemp, Campinas, v.2, nov. 2006.

GREWAL, D.; LEVY, M. Marketing. 2 ed. São Paulo: Bookman, 2011.

GULLO, J.; PINHEIRO, D. Fundamentos de marketing: suporte às estratégias de negócios das empresas. São Paulo: Atlas, 2011.

KOTLER, P. Administração de marketing: análise, planejamento, implementação e controle. 5. ed. São Paulo: Atlas, 1998.

; KELLER, K. L. Administração de marketing. 12 ed. São Paulo: Pearson Prentice hall, 2006.

LEYSER, M. Texturas visuais em embalagens para cosméticos femininos: estudo de caso de um produto no mercado. 126 f. Dissertação (Mestrado em Design) Universidade Federal do Rio Grande do Sul, Porto Alegre, 2012.

MACARRÃO JUNIOR, L. Importância do uso de mock-ups e de técnicas de prototipagem e ferramental rápido no processo de desenvolvimento de produto na indústria automotiva. $157 \mathrm{f}$. Trabalho de Conclusão de Curso (Mestrado Profissionalizante em Engenharia Automotiva) - Escola Politécnica, Universidade de São Paulo, São Paulo, 2004.

MCLLVEEN, H. Product Development and the Consumer: the Reality of Managing Creativity. Nutrition and Food Science, n. 6, pp. 26-30, 1994.

MOREIRA, D. A. Administração da Operação e Produções. 5 ed. São Paulo: Pioneira, 2000.

MOWER, J. C.; MINOR, M. S. Comportamento do consumidor. São Paulo: Prentice Hall, 2003.

REZENDE, F. A.; ALVES, L. F. P.; SCHEFFER, R. C.; SILVA, V. L. da. Projeto de produto e o desenvolvimento de um novo produto: Minipizza doce congelada. Trabalho de Disciplina (Graduação em Engenharia de Produção Agroindustrial) - 
Departamento de Engenharia de Produção, Campus de Campo Mourão, Universidade Estadual do Paraná, UNESPAR, Campo Mourão/PR, 2013.

RICHERS, R. O enigmático mais indispensável consumidor: teoria e prática. Revista da Administração, jul./set. de 1984.

RODRIGUES, M. A.; JUPI, V. S. O comportamento do consumidor - Fatores que influenciam em sua decisão de compra. Revista de Administração Nobel, no 3, p. 59-70, jan./jun. 2004.

SCARABOTO, D. Comunidades virtuais como grupos de referência nos processos decisórios do consumidor. Dissertação (Mestrado em Administração) Universidade Federal do Rio Grande do Sul, UFRS. Porto Alegre, 2006.

SCHIFFMAN, L. G.; KANUK, L. L. Comportamento do consumidor. 6. ed. Rio de Janeiro: LTC, 2000.

SHETH, N. J.; MITTAL, B.; NEWMAN, I. B. Comportamento do cliente: indo além do comportamento do consumidor. São Paulo: Atlas, 2001.

SHIMOYAMA, C.; ZELA, D. R. Administração de Marketing. In: BARBOSA, E. F. Marketing. Curitiba: Faculdades Bom Jesus, 2002. Cap. 1, p. 1-18.

SOUSA, V. M. B. Fatores que influenciam o comportamento de compra dos consumidores em uma empresa varejista de móveis e eletrodomésticos em Picos-PI. Monografia (Bacharelado em Administração). Picos/PI: Universidade Federal do Piauí, 2012.

SOUZA, A. R. C.; FARIAS, J. M. P.; NICOLUCI, T. C. Principais fatores que influenciam os consumidores universitários a frequentarem bares noturnos na cidade de Presidente Prudente. Monografia (Bacharelado em Administração). Presidente Prudente, SP: UNITOLEDO/FIAETPP, 2005.

STONE, B. Marketing direto. 4 ed.São Paulo: Nobel, 1992.

TEIXEIRA, J. C. I. Fatores que influenciam o comportamento do consumidor. Dissertação (Pós-Graduação em Gestão estratégica) - Universidade Cândido Mendes. Rio de Janeiro, 2010.

ZUIN, L. F. S et al. Emprego de testes de mercado no processo de desenvolvimento de produto na industria alimentícia: uma análise exploratória. 
In: XI SIMPÓSIO DE ENGENHARIA DE PRODUÇÃO, 2004, Bauru. Anais... Bauru: 2004.

\section{Apêndice 1}

Figura 2 - Detalhamento do processo para a especificação da Apresentação Física de Produtos em Projeto de Produtos alimentícios

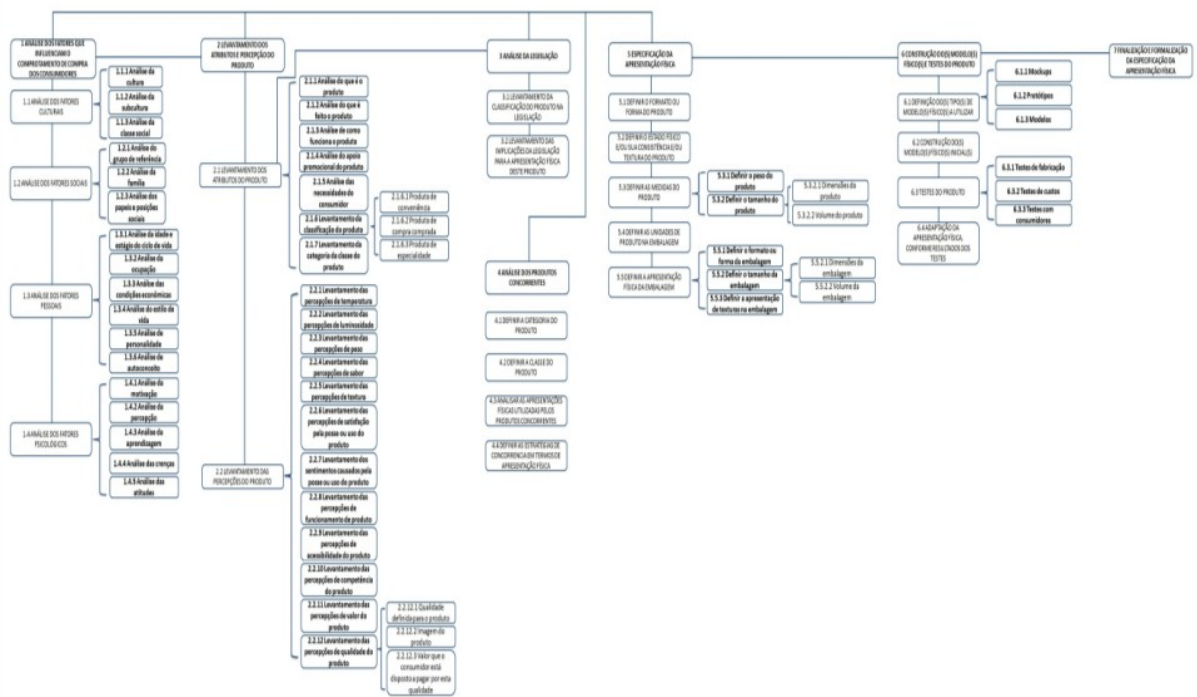

Fonte: Elaborado pelos autores, com informações obtidas em Boiko (2013)

Recebido: 20 dez. 2016

Aprovado: 14 fev. 2017

DOI: 10.3895/gi.v13n1.5242

Como citar:

BOIKO, T. J. P. et al. Apresentação física de produtos em projetos de produtos alimentícios: proposição de um processo para a especificação. R. Gest. Industr., Ponta Grossa, v. 13, n. 1, p. 176-193, jan./mar. 2017.

Disponível em: <https://periodicos.utfpr.edu.br/rgi>. Acesso em: XXX.

Correspondência:

Thays J. Perassoli Boiko

Universidade Estadual do Paraná - Campus de Campo Mourão. Avenida Comendador Noberto Marcondes, n. 733, Centro, Campo Mourão, Paraná, Brasil.

Direito autoral: Este artigo está licenciado sob os termos da Licença Creative Commons-Atribuição 4.0

Internacional.

(c) (1) 